\title{
Inhibitory of EV-A71 virus-induced apoptosis by ZVAD through ROS mediated signaling pathways
}

\author{
Tiantian XU ${ }^{1, \#}$; Ruilin ZHENG ${ }^{1, \#}$; Danyang CHEN ${ }^{1, *}$; HaiYang CHEN ${ }^{2}$; MingQi ZHAO Z Min GUO $^{1}$; Yi CHEN ${ }^{1}$; \\ Changbing WANG ${ }^{1}$; Lu KUANG ${ }^{1}$; Yinghua $\mathrm{LI}^{1, *}$; Bing $\mathrm{ZHU}^{1, *}$ \\ 1 Center Laboratory, Guangzhou Women and Children's Medical Center, Medical University, Guangzhou, 510120, China \\ 2 Nanfang Hospital, Southern Medical University, Guangzhou, 510515, China
}

Key words: ZVAD, EV-A71 virus, ROS, Apoptosis

\begin{abstract}
Emerging evidence that Enterovirus A 71 (EV-A71) infection closely related to apoptosis. The ZVAD is a caspase inhibitor that can prevent apoptosis. The aims of this project were to evaluate the mechanism of the ZVAD inhibited EV-A71 virus and to provide experiment basis for finding new antiviral drugs. In this study, after treated with ZVAD in EV-A71 infected Vero cells, the viral replication was reduced, and the cell viability was higher than EV-A71 group. Additionally, ZVAD decreased the cell apoptosis and the level of inflammatory cytokines induced by EV-A71 in the infected Vero cells. ZVAD inhibited cell apoptosis by regulating ROS mediated signaling pathway and inflammation cytokines to achieve antiviral.
\end{abstract}

\section{Introduction}

Hand, foot, and mouth disease (HFMD) is commonly affects children under the age of five in children (Li et al., 2017b; Zeng et al., 2019). HFMD is mainly caused by enterovirus A71 (EVA71), coxsackievirus 16 (Cox16), CV-A6 and CV-A10. Most of these infections are exhibit self-limiting characterized by skin rash and sores on hands, feet and in the mouth with or without fever. However, some patients infected with EVA71 virus can develop severe central nervous system damage or cardiopulmonary complications or die (Cao et al., 2019; Li et al., 2019). EV-A71 was first isolated from patients with a nervous system disease in California in 1969 (Wang et al., 2017b). Since then, outbreaks of HFMD have been documented in countries of the Asia (Xiao et al., 2018; Xing et al., 2014; Xu et al., 2019), including Singapore, Malaysia, China and so on, which takes huge losses to social economy. Although the vaccine of EV-A71 has been approved for the prevention of hand, foot and mouth disease, it is still facing various problems: time-consuming, laborious and low antibody titer (Chong et al., 2015; Imura et al., 2020a; Imura et al., 2020b). Currently, the

\footnotetext{
*Address correspondence to: Yinghua Li, liyinghua@gzhmu.edu.cn; Bing Zhu, zhubing@gzhmu.edu.cn

${ }^{\#}$ These authors contributed equally to this work

Received: 04 June 2021; Accepted: 14 August 2021
}

pathogenicity of EV-A71 is not entirely understood and there are no effective antiviral treatments to treat EV-A71induced HFMD.

Apoptosis is a regulated physiological process involved in cell death (Raymond et al., 2004). The process of apoptosis has been implicated in the pathogenesis of many infectious diseases (Mehrbod et al., 2019; Qu et al., 2017; Ravindra et al., 2008). EV-A71 is one of such virus, which has been shown to cause apoptosis in target cells (Bai et al., 2019; Li et al., 2020b). In particular, the apoptosis of the nerve cells may be associated with severe EV-A71. In addition, it was reported that the EV-A71 induces apoptosis by promoting ROS generation and depending on caspase families (Li et al., 2020b). The compound Z-Val-Ala-Asp-fluoromethylketone (zVAD-fmk, ZVAD), a widely used broad-caspase inhibitor, can prevents apoptosis in many different cell types (Hotchkiss et al., 2000; Li et al., 2000; Zeng et al., 2019). It was reported that ZVAD reduced hepatocarcinogenesis in a transgenic murine model of hepatitis $\mathrm{B}$ virus. Moreover, ZVAD inhibited Sindbis virus-induced cell death. It was also reported that ZVAD can reduced apoptosis in virus infected cells. However, that was remained unclear how ZVAD modulate the antiviral activity in Vero cells.

Herein, the aim of this study was to evaluate the mechanism of ZVAD on inhibition of EV-A71 infection. First, we measured the cell toxicity of ZVAD via CCK8; then, the antiviral effect of ZVAD was detected by Western blot and QRT-PCR. Finally, the therapeutic mechanisms of 
ZVAD were examined by flow cytometry, dichlorofluorescein (DCF) and western blot. This paper provides a theoretical basis for the treatment of EV-A71 by ZVAD.

\section{Materials and Methods}

\section{Materials}

African green monkey kidney epithelial (Vero) cells were purchased from the American Type Culture Collection (ATCC, CRL-1586TM and THB-11TM). Dulbecco's modified Eagle's medium (DMEM) and fetal bovine serum (FBS) were acquired from Gibco. Cell Counting Kit-8 (CCK8) and Enhanced chemiluminescence (ECL) detection kit were purchased from Beyotime, China. Enterovirus A71 (EV-A71) is a C4 genotype Guangdong strain (GenBank Accession No. FJ439769.1) stored at our laboratory. The broad caspase inhibitor ZVAD was obtained from MCE MedChemExpress, China. The reverse transcription polymerase chain reaction (RT-PCR) kit and the agarose gel DNA extraction kit were all purchased from Takara, Japan. The EV-A71 VP1 monoclonal antibody (ab169442) was obtained from Abcam, UK. ERK (\#4695S), Caspase3 (\#9662S), PARP (\#9542S), $\beta$-actin antibody (\#3700S) were purchased from Cell Signaling Technology.

\section{Viruses and infection}

Vero cells were cultured in DMEM medium, supplemented with $10 \% \mathrm{FBS}$ and $100 \mathrm{U} / \mathrm{mL}$ penicillin at $37^{\circ} \mathrm{C}$ in $5 \% \mathrm{CO}_{2}$ humidified atmosphere. Then the Vero cells were used for infection. EV-A71 were propagated and its 50\% tissue culture infective dose (TCID50) were determined using the Reed-Muench method (Przybylski et al., 2015). EV-A71 was used in this study at a final titer of 100 TCID50 and the virus infection was carried out as described previously (Xu et al., 2019).

\section{Cell viability assay}

Vero cells at the density of $5 \times 10^{4}$ were split into 96-well plates overnight. After that, the media were removed, and cells were mock incubation or incubation with EV-A71 (100 TCID50) for $2 \mathrm{~h}$ (Zhu et al., 2017). Thereafter, the cells incubated with or without different concentrations of ZVAD for $48 \mathrm{~h}$. Then the cells were treated with $10 \mu \mathrm{L}$ CCK8 reagents for $30 \mathrm{~min}$ at $37^{\circ} \mathrm{C}$ in the dark. The absorbance was measured in a microplate reader at $450 \mathrm{~nm}$. Cell viabilities were expressed as the ratio of the experimental group to the negative control.

\section{Quantitative Real-Time PCR (QRT-PCR)}

The total RNA was extracted by the Trizol reagent according to the manufacturer's instructions. The RNA of EV-A71 VP1 were detected by real-time PCR using SYBR green (Li et al., 2019). Amplification was performed on an Applied Biosystems (ABI) 7500 instrument at $98^{\circ} \mathrm{C}$ for $8 \mathrm{~min}$, followed by 40 cycles at $98^{\circ} \mathrm{C}$ for $15 \mathrm{~s}, 54^{\circ} \mathrm{C}$ for $20 \mathrm{~s}$ and $68^{\circ} \mathrm{C}$ for $2 \mathrm{~min}$, and a final extension step at $68^{\circ} \mathrm{C}$ for $5 \mathrm{~min}$. QRT-PCR reactions were performed in triplicate, results were reported as fold change using the $\Delta \Delta \mathrm{CT}$ (CT is threshold cycle) method. Primer sequences are listed in Table 1.

\section{TABLE 1}

Primers used in this study

\begin{tabular}{ll}
\hline Genes & Sequence \\
\hline VP1 F & 5'-CAAATGCGTAGAAAGGTGGAG-3' \\
VP1 R & 5'-GGAGCAACTGTGGGACAACT-3' \\
B-actin F & 5'-GACTTCGAGCAGGAGATGGG-3' \\
B-actin R & 5'-CGAGGAAGGAAGGCTGGAAG-3' \\
\hline
\end{tabular}

Western blot analysis

The effects of ZVAD on the expression levels of apoptotic proteins and VP1 were detected by western blotting referring to previous reported (Wang et al., 2017a). Briefly, The Vero Cells were harvested using RIPA Lysis Buffer and the total protein samples concentration were determined by BCA assay. The protein samples were loaded on SDSpolyacrylamide gel for electrophoresis (SDS-PAGE) and transferred onto PVDF transfer membranes. Membranes were blocked with 5\% milk and then incubated with primary antibody overnight at $4^{\circ} \mathrm{C}$. After washing by TBST, the membranes were incubated with secondary antibody. Then the proteins were detected by ECL detection kit.

\section{Annexin-V-FITC staining assay}

Vero cells were cultured in 6-well plates for $24 \mathrm{~h}$. After the cells reached about $80 \%$ confluence, the EV-A71 were incubated as mentioned above followed by ZVAD $25 \mu$ mol. After $48 \mathrm{~h}$, the cells were collected and fixed with $70 \%$ ethanol at $4^{\circ} \mathrm{C}$ for $6 \mathrm{~h}$. Then the cells were staining with Annexin V-FITC/ propidium iodide (PI) and were detected by flow cytometry as previously described (Bai et al., 2020). All flow cytometry was performed on a $\mathrm{BD}$ flow cytometer. The data were analyzed by the FlowJo software (Treestar, USA).

ROS levels analysis

It has been reported that ROS was a vital regulator of cell signaling pathways triggered by virus (Li et al., 2020a; Qian et al., 2018). In order to investigate whether ZVAD could trigger ROS-mediated apoptosis, the ROS level was detected by Fluorescent dye 2,7-dichlorofluorescein-diacetate (DCFCH-DA) fluorescence assay (Li et al., 2017a). After Vero cells treated with EV-A71 followed by ZVAD, Cells were washed twice and loaded with DCFH-DA $(10 \mu \mathrm{mol} / \mathrm{L})$ and incubated at $37^{\circ} \mathrm{C}$ for $20 \mathrm{~min}$. The fluorescence of $\mathrm{DCFH}$ was measured on inverted fluorescence microscope.

Cytokine analysis

The levels of IL- 8 in the cell culture media were measured by flow cytometry refer to previously reported (Zhao et al., 2019). In brief, the cell culture media were collected and centrifuged at $3000 \mathrm{rpm}$ for $5 \mathrm{~min}$. Then $45 \mu \mathrm{L}$ samples were mixed with $45 \mu \mathrm{L}$ fluorescent bead carrying surface-immobilized antibodies. Then the sample were measured using flow cytometry (BD Company, USA) and the data were acquired through BD FACSDiva Software. The concentrations were calculated by standard curves using FCAP Array 3.0 analysis software. 


\section{Statistical analysis}

All the data in this study are means \pm the standard deviation (SD). Statistical significance was evaluated as follows: ${ }^{\star} P<$ $0.05,{ }^{* *} P<0.01$ or ${ }^{\star * *} P<0.001$.

\section{Results}

\section{ZVAD enhances cells viabilities in vitro}

To detect the anti-EV-A71 activities of the ZVAD compounds, the Cytopathic effect (CPE) induced by EVA71 in Vero cells were observed. First, the cell toxicity of ZVAD we measured by CCK8 assay and found that ZVAD had no toxic to cells within the scope in the manual. Next, we evaluated the antiviral activities of ZVAD $(25 \mu \mathrm{mol} / \mathrm{L})$ in Vero cells by CCK8 assay. As was seen in Fig. 1A, Vero cells infected with EV-A71 showed cytoplasmic shrink age and loss of cell-cell contract, however, the cell morphology was appeared healthy by treatment with ZVAD. As shown in Fig. $1 B$, the viability of EV-A71 infected Vero cells were showed to $53.56 \%$, but the cell viability was increased to $83.7 \%$ by ZVAD (Fig. 1B).

$Z V A D$ reduced the $R N A$ and protein synthesis of EV-A71 in Vero To further investigate the effects of ZVAD on the virus, the RNA and protein synthesis of EV-A71 were detected by QRT-PCR and Western blot respectively. The level of VP1 RNA measured by QRT-PCR assay were significantly decreased in ZVAD treated group than ZVAD un-treated group (Fig. 2A). Similarly, the level of VP1 proteins detected by Western blot were also decreased in ZVAD treated group than ZVAD un-treated group, as shown in Fig. 2B. Our data showed that the viral replication and synthesis were reduced in Vero cells treated with ZVAD.

ZVAD suppressed the apoptosis induced by EV-A71

It was reported that ZVAD inhibited Sindbis virus-induced cell death. Whereas, the mechanism of ZVAD reduces apoptosis in EV-A71 infected Vero cells remains unclear. Annexin-V/PI double staining is an effective method for examining apoptosis that was detected through flow cytometry. The apoptosis rate of Vero cells infected with EVA71 were $60.8 \%$. Nevertheless, the apoptosis was significantly reduced in ZVAD treated group, and the rate of cell apoptosis were reduced to $25.69 \%$, as shown in Fig. $3 \mathrm{~A}$. It was reported that EV-A71 infection triggers mitochondrial ROS, furthermore ROS promoted the EV-A71-induced apoptosis and inflammation. In order to investigate whether ZVAD restrain apoptosis induced by EV-A71 virus through ROS, we monitored intracellular ROS level by inspecting the fluorescence intensity of dichlorofluorescein (DCF). As shown in Fig. 3B, we observed strong fluorescent intensity of DCF in EV-A71 infected Vero cells. Fluorescence was turned weak in ZVAD treated with cells. These results revealed that ZVAD restrained ROS generation and suppressed the EVA71 virus proliferation in Vero cells.

\section{ZVAD regulatory cytokines}

IL- 8 is a chemokine and can be produced by multihistocytes. It was reported that the serum levels of IL- 8 significantly increased in EV71 critical infection compared to mild cases and severe cases. To characterize the effect of ZVAD on inflammation in EV-A71 infected Vero cells, the levels of the culture medium inflammatory cytokines were detected by flow cytometry. In this study, it was showed that ZVAD treatment could decrease the level of IL8 (3.28 pg/mL) compared to the virus control $(5.21 \mathrm{pg} / \mathrm{mL})$ (Fig. 4). These findings suggest that ZVAD exerts its anti-inflammation effect by accommodate inflammation cytokines.

Detection of apoptosis signaling pathway related proteins To detect the effect of ZVAD in EV-A71 infected cells, the apoptosis proteins were detected via Western Blotting (Wang et al., 2017a). Caspase3 is an important component of apoptosis and is an effector caspase involved in
(A)

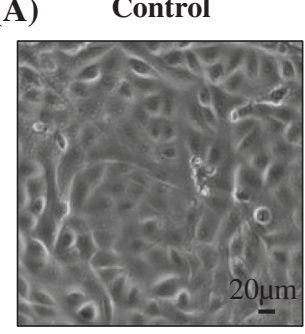

ZVAD

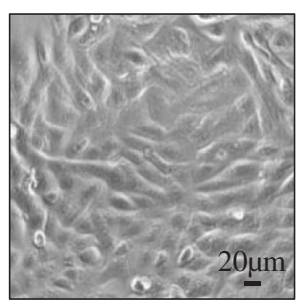

EV-A71

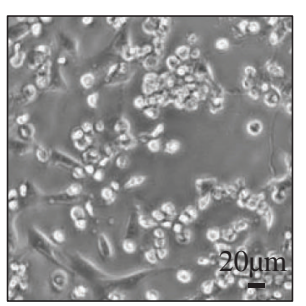

EV-A71+ZVAD

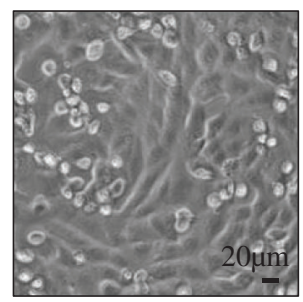

(B)

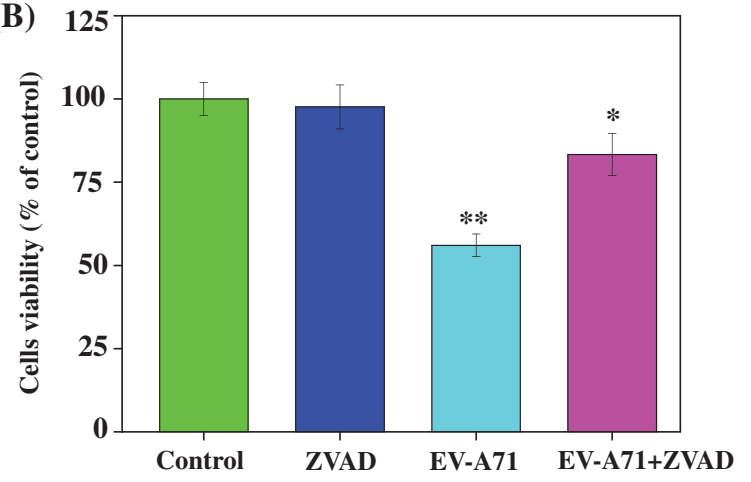

FIGURE 1. The cells viability of ZVAD. (A) ZVAD reduced the EVA71 induced CPE in Vero cells. Cells were examined using a microscopy ( $\times 20)$. (B) The CCK8 assay of ZVAD treated EV-A71 infected Vero cell. The concentration of ZVAD were 25 $\mu \mathrm{mol} / \mathrm{L}$. Control, Vero without any treatment; ZVAD, Vero cells treated with ZVAD; EV-A71, Vero infected with EV-A71; EV-A71+ZVAD, EVA71 infected Vero cells treated with ZVAD. 

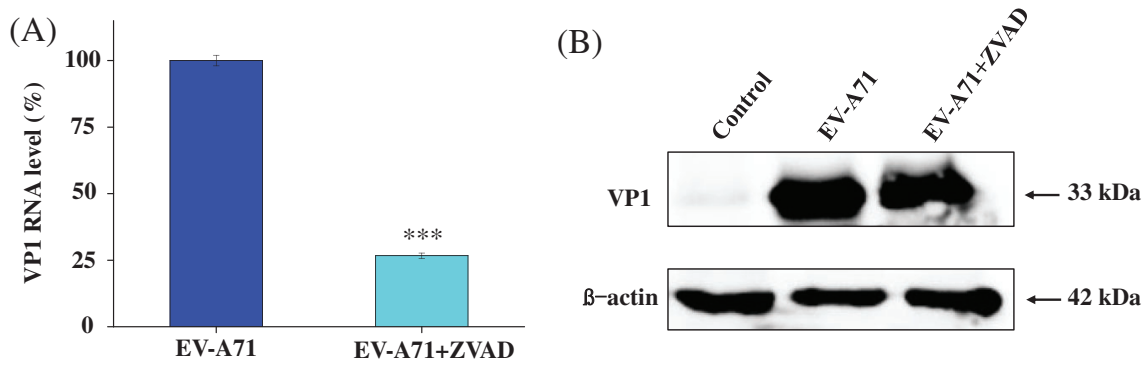

FIGURE 2. Suppression of VP1 mRNA and protein expression in ZVAD treated Vero cells. (A) Suppression of VP1 mRNA levels was quantified by Q-PCR. (B) The expression of VP1 was evaluated by Western blotting. ${ }^{* * *} P<0.001,{ }^{* \star} P<0.01,{ }^{\star} P<0.05$.

(A) Control
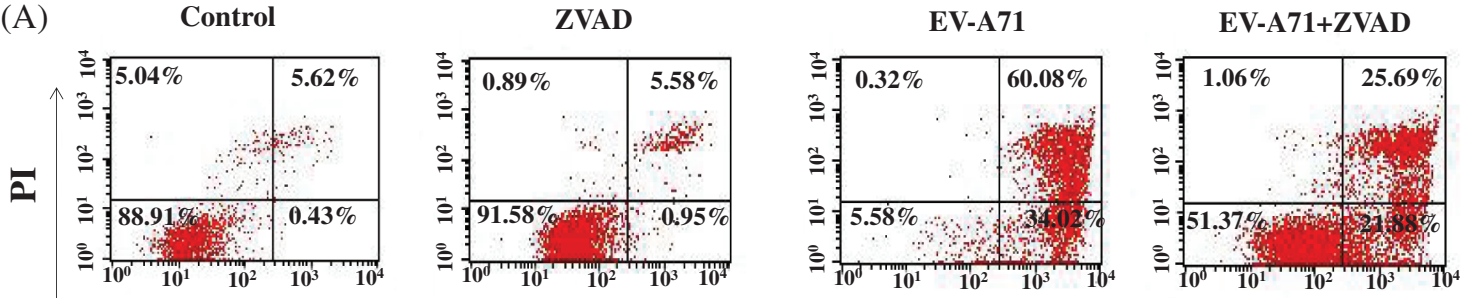

Annexin V-FITC

(B)

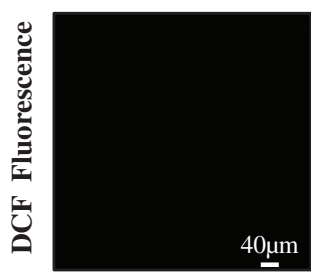

ZVAD

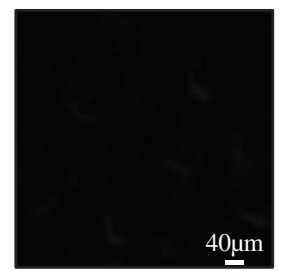

EV-A71

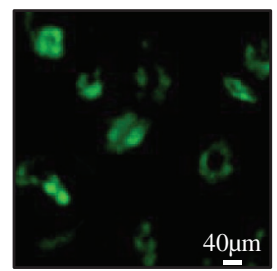

EV-A71+ZVAD

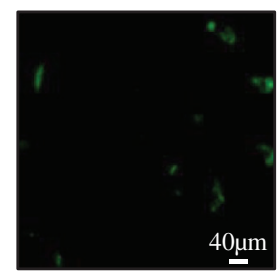

FIGURE 3. ZVAD suppressed the apoptosis induced by EV-A71 in Vero cells. (A) Annexin V-FITC and PI flow cytometry was performed to detect the number of apoptosis in EV-A71-infected Vero cells. The upper right quadrant of every plot represents late dead cells. (B) DCF fluorescence intensity assay was conducted to investigate the ROS production among control, ZVAD, EV-A71, ZVAD+ EV-A71. Control, Vero cells without any treatment; ZVAD, Vero cells treated with ZVAD; EV-A71, Vero cells infected with EV-A71; EV-A71+ZVAD, EVA71 infected Vero cells treated with ZVAD.

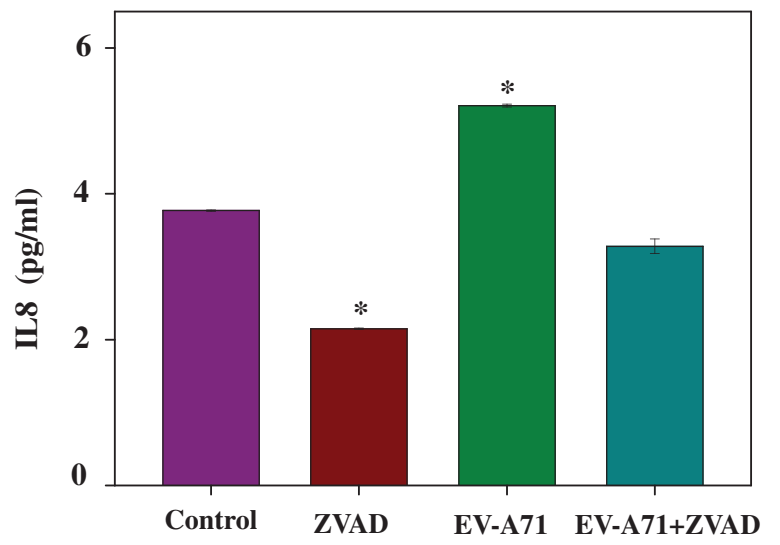

FIGURE 4. Flow cytometry assay was used to study the expression level of the inflammatory cytokines. ZVAD decreased the expression of IL8 in EV-A71 infected Vero cells. ${ }^{* *} P<0.001,{ }^{* *} P<0.01$ ${ }^{\star} P<0.05$, compared with the EV-A71 infected model. Control, Vero cells without any treatment; EV-A71, Vero cells infected with EV-A71; ZVAD, Vero cells treated with $25 \mu \mathrm{mol} / \mathrm{L}$ ZVAD.

proteolytic cleavage of important proteins like PARP, as shown in Fig. 5A, the level of caspase-3 expression was down regulated, and the PARP was up regulated with treatment of ZVAD. This suggesting that the apoptosis of Vero cells induced by EV-A71 were effectively resisted when treated with ZVAD. Besides, the level of P-ERK was increased in ZVAD treated group than no ZVAD treated group (Fig. 5A). Taken together, the results indicate that ZVAD depressed EV-A71-induced host-cells apoptosis by ROS mediated signaling pathways (Fig. 5B).

\section{Discussion}

Severe hand-foot and mouth disease (HFMD) caused by enterovirus 71 (EV-A71) is often accompanied by central nervous system symptoms such as encephalitis ( $\mathrm{Li}$ et al., 2020b). The specific mechanism for severe neurological complications caused by EV-A71 infection is still unclear, and there is no specific antiviral treatment currently. Therefore, the pathogenesis of EV-A71 infection has received extensive attention in the field of medicine and biology.

Our dates showed that viral replication was reduced when EV-A71 infected Vero cells treated with ZVAD. Meanwhile, EV-A71 infected Vero cells were exhibit higher cells viability in the presence of ZVAD. Additionally, apoptosis, ROS and inflammation induced by EV-A71 infection were decreased by ZVAD. These findings strongly suggest that ZVAD exerts its regulatory effect on EV-A71 replication by inducing ROS production, apoptosis, and inflammation. 
(A)

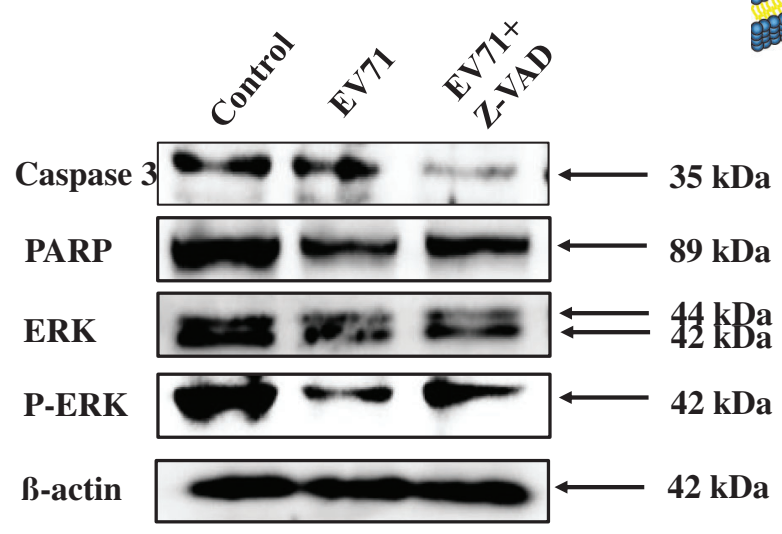

(B)

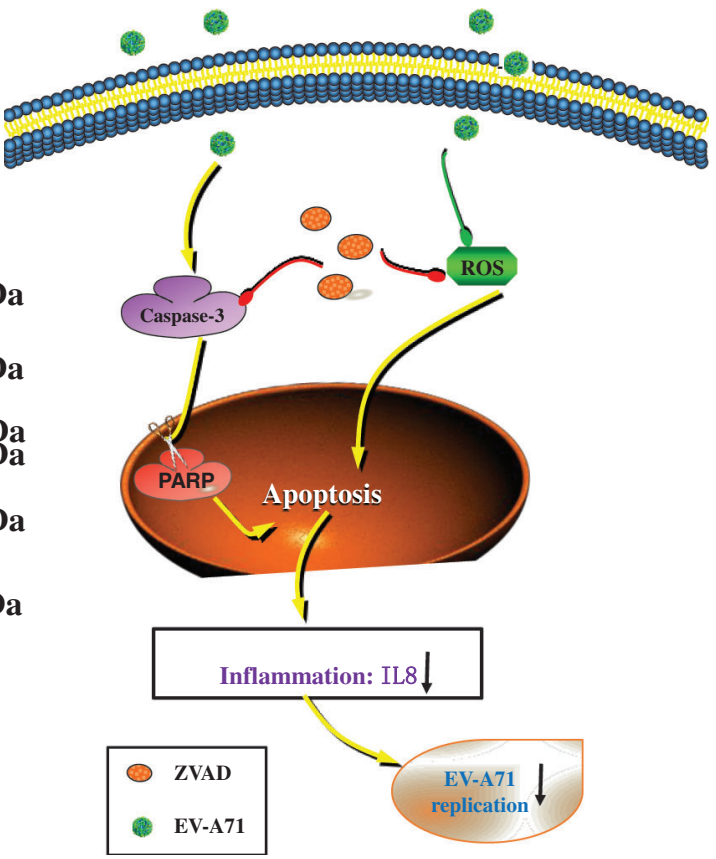

FIGURE 5. Signaling pathways participated in the inhibition of EV-A71 by ZVAD. Cells were uninfected or infected with $100 \mathrm{TCID} 50 \mathrm{EV}-\mathrm{A} 71 \mathrm{for} 2 \mathrm{~h}$. The cells were then treated with ZVAD for $48 \mathrm{~h}$. Then cells were harvested and proteins were examined by western blot. The caspase 3 were inhibited and the phosphorylation of ERK were increased in ZVAD treated Vero cells (A). Sketch of caspase 3 and ROS signaling pathways (B). Control, Vero cells without any treatment; EV-A71, Vero cells infected with EV-A71; EV-A71+ZVAD, EV-A71 infected Vero cells treated with ZVAD.

Caspase activation is a central induction of apoptosis. The caspase 8 and caspase 9 are called "initiator caspases" were activated first, while the caspase 3 which are called "executioner caspases" mediated the cell death (Chen et al., 2021; Lin et al., 2021). Studies have shown that caspases mediated apoptosis play a key role in neuronal injury caused by EV-A71 infection (Song et al., 2018). Meanwhile, ZVAD is a broad-caspase inhibitor can prevent apoptosis in many different cell types. Our result shows that the apoptosis was inhibited by ZVAD through reduce caspase 3 and the reduction of PARP. It was also reported that the ZVAD have an anti-inflammation effect (Liu et al., 2016). However, it was remained unknown how ZVAD modulate the anti-EV-A71 activity in Vero cells.

In addition, several studies have also demonstrated that EV-A71 infection leads to production of ROS (Chan et al., 2021; Cheng et al., 2014; Huang et al., 2020; Wu et al., 2019), which may be involved in enhanced cellular apoptosis and viral replication (Ho et al., 2008). In this study, we have revealed that ZVAD did reduce the activity of ROS in-A71 infected Vero cells. The induction of apoptosis in EV-A71 infected Vero cells can be inhibited by ZVAD via inhibiting caspase and ROS activity.

IL-8 is a chemokine and can be produced by multihistocytes. The main role of IL- 8 is to attract and activate neutrophils therefore it could produce inflammation in the body. It was reported that the serum levels of IL-8 significantly increased in EV71 critical infection compared to mild cases and severe cases. Besides, ZVAD can regulate inflammatory cytokines by necropotosis in virus infected cells (van den Berg et al., 2015). In this study, IL8 were decreased in EV-A71 infected Vero cells treated with ZVAD compared to mock virus infection. Whether ZVAD directly regulated the inflammatory cytokines caused by the virus needs to be further studies.
It was known that ERK can be activated by EV-A71 infection in various cell types and inhibition of ERK signaling pathway by specific siRNAs has been found to impair EV-A71 replication. However, we found that viral replication was decreased when P-ERK was increased in Vero cells treated by ZVAD. This may be because the activation and function of ERK varied in differently time and different cell lines. In addition, ZVAD may have different effects in the same signaling pathway in different cell lines.

\section{Conclusion}

Our data strongly support that ZVAD can effectively anti-EVA71 virus proliferation in infected Vero cells. ZVAD reduced cell apoptosis by inhibiting the activity of caspase and ROS generation. Whether ZVAD directly reduced apoptosis by ROS signaling pathways required further investigation. In the future, we will perform in vivo experiments to better understand these mechanisms.

Availability of Data and Material: All data generated or analyzed during this study are included in this published article and its supplementary information files.

Author's Contribution: Study design: BZ; experiments carry out: LK, RLZ, MG and DYC; data collection HYC, CBW; analysis of results: $\mathrm{CBW}$ and $\mathrm{MQZ}$; draft manuscript preparation: YHL and TTX. All authors reviewed the final version of the manuscript.

Ethics Approval: This project was approved by the Ethics Committee of Guangzhou Women and Children Medical Center (Approval No. 2017021803).

Funding Statement: This work was supported by the fund from Open Project of Guangdong Key Laboratory of Marine 
Materia Medica (LMM2020-7), the fund from Guangzhou Institute of Pediatrics/Guangzhou Women and Children's Medical Center (Nos. Pre-NSFC-2019-016, YIP-2019-059, IP-2019-019), the Project of Traditional Chinese Medicine Bureau of Guangdong Province (No. 20192075), the technology planning projects of Guangdong province (No. 202102010202). The Guangdong Natural Science Foundation (2020A1515110648), the Open Fund of Guangdong Provincial Key Laboratory of Functional Supramolecular Coordination Materials and Applications (2020A03).

Conflicts of Interest: The authors declare that they have no competing interests.

\section{References}

Bai J, Chen X, Liu Q, Zhou X, Long JE (2019). Characteristics of enterovirus 71-induced cell death and genome scanning to identify viral genes involved in virus-induced cell apoptosis. Virus Research 265: 104-114.

Bai Z, Zhao X, Li C, Sheng C, Li H (2020). EV71 virus reduces Nrf2 activation to promote production of reactive oxygen species in infected cells. Gut Pathogens 12: 22.

Cao L, Zhang X, Yuan S, Cheng K, Zhang X (2019). Autophagy induced by enterovirus 71 regulates the production of IL- 6 through the p38MAPK and ERK signaling pathways. Microbial Pathogenesis 131: 120-127.

Chan L, Chen X, Gao P, Xie J, Zhang Z et al. (2021). Coordinationdriven enhancement of radiosensitization by black phosphorus via regulating tumor metabolism. ACS Nano 15: 3047-3060.

Chen M, Huang X, Lai J, Ma L, Chen T (2021). Substituent-regulated highly X-ray sensitive Os (VI) nitrido complex for lowtoxicity radiotherapy. Chinese Chemical Letters 32: 158-161.

Cheng ML, Weng SF, Kuo CH, Ho HY (2014). Enterovirus 71 induces mitochondrial reactive oxygen species generation that is required for efficient replication. PLoS One 9: e113234. DOI 10.1371/journal.pone.0113234.

Chong P, Liu CC, Chow YH, Chou AH, Klein M (2015). Review of enterovirus 71 vaccines. Clinical Infectious Diseases 60: 797-803. DOI 10.1093/cid/ciu852.

Ho HY, Cheng ML, Weng SF, Chang L, Yeh TT et al. (2008). Glucose-6-phosphate dehydrogenase deficiency enhances enterovirus 71 infection. Journal of General Virology 89: 2080-2089. DOI 10.1099/vir.0.2008/001404-0.

Hotchkiss RS, Chang KC, Swanson PE, Tinsley KW, Hui JJ et al. (2000). Caspase inhibitors improve survival in sepsis: A critical role of the lymphocyte. Nature Immunology 1: 496501. DOI $10.1038 / 82741$.

Huang W, He L, Ouyang J, Chen Q, Liu C et al. (2020). Triangleshaped tellurium nanostars potentiate radiotherapy by boosting checkpoint blockade immunotherapy. Matter 3: 1725-1753. DOI 10.1016/j.matt.2020.08.027.

Imura A, Sudaka Y, Takashino A, Tamura K, Kobayashi K et al. (2020a). Development of an enterovirus 71 vaccine efficacy test using human scavenger receptor B2 transgenic mice. Journal of Virology 94: 251. DOI 10.1128/JVI.01921-19.

Imura A, Sudaka Y, Takashino A, Tamura K, Kobayashi K et al. (2020b). Development of an enterovirus 71 vaccine efficacy test using human scavenger receptor B2 transgenic mice. Journal of Virology 94: 251. DOI 10.1128/JVI.01921-19.
Li H, Bai Z, Li C, Sheng C, Zhao X (2020a). EV71 infection induces cell apoptosis through ROS generation and SIRT1 activation. Journal of Cellular Biochemistry 121: 4321-4331. DOI 10.1002/jcb.29628.

Li H, Bai Z, Li C, Sheng C, Zhao X (2020b). EV71 infection induces cell apoptosis through ROS generation and SIRT1 activation. Journal of Cellular Biochemistry 121: 4321-4331. DOI $10.1002 / j \mathrm{jcb} .29628$.

Li M, Ona VO, Guegan C, Chen M, Jackson-Lewis V et al. (2000). Functional role of caspase- 1 and caspase- 3 in an ALS transgenic mouse model. Science 288: 335-339. DOI 10.1126/science.288.5464.335.

Li Y, Lin Z, Guo M, Xia Y, Zhao M et al. (2017a). Inhibitory activity of selenium nanoparticles functionalized with oseltamivir on $\mathrm{H} 1 \mathrm{~N} 1$ influenza virus. International Journal of Nanomedicine 12: $5733-5743$. DOI $10.2147 / \mathrm{IJN}$

Li Y, Lin Z, Xu T, Wang C, Zhao M et al. (2017b). Delivery of VP1 siRNA to inhibit the EV71 virus using functionalized silver nanoparticles through ROS-mediated signaling pathways. RSC Advances 7: 1453-1463. DOI 10.1039/C6RA26472G.

Li Y, Xu T, Lin Z, Wang C, Xia Y et al. (2019). Inhibition of enterovirus $A 71$ by selenium nanoparticles interferes with JNK signaling pathways. ACS Omega 4: 6720-6725. DOI 10.1021/acsomega.8b03502.

Lin W, Liu H, Chen L, Chen J, Zhang D et al. (2021). Pre-clinical MRI-guided intravesical instillation theranosis of bladder cancer by tumor-selective oxygen nanogenerator. Nano Today 38: 101124. DOI 10.1016/j.nantod.2021.101124.

Liu M, Shi L, Zou X, Zheng X, Zhang F et al. (2016). Caspase inhibitor zVAD-fmk protects against acute pancreatitis-associated lung injury via inhibiting inflammation and apoptosis. Pancreatology 16: 733-738. DOI 10.1016/j.pan.2016.06.002.

Mehrbod P, Ande SR, Alizadeh J, Rahimizadeh S, Shariati A et al. (2019). The roles of apoptosis, autophagy and unfolded protein response in arbovirus, influenza virus, and HIV infections. Virulence 10: 376-413.

Przybylski M, Borysowski J, Jakubowska-Zahorska R, WeberDąbrowska B, Górski A (2015). T4 bacteriophage-mediated inhibitionof adsorption and replication of human adenovirus in vitro. Future Microbiology 10: 453-460.

Qian M, Tan HM, Yu N, Wang T, Zhang Q (2018). Inactivated sendai virus induces ROS-dependent apoptosis and autophagy in human prostate cancer cells. Biomedical and Environmental Sciences 31: 280-289.

Qu X, Ding X, Duan M, Yang J, Lin R et al. (2017). Influenza virus infection induces translocation of apoptosis-inducing factor (AIF) in A549 cells: Role of AIF in apoptosis and viral propagation. Archives of Virology 162: 669-675.

Ravindra PV, Tiwari AK, Ratta B, Chaturvedi U, Palia SK et al. (2008). Induction of apoptosis in Vero cells by Newcastle disease virus requires viral replication, de-novo protein synthesis and caspase activation. Virus Research 133: $285-290$.

Raymond MA, Desormeaux A, Laplante P, Vigneault N, Filep JG et al. (2004). Apoptosis of endothelial cells triggers a caspase-dependent anti-apoptotic paracrine loop active on VSMC. FASEB Journal 18: 705-707. DOI 10.1096/fj.030573fje.

Song F, Yu X, Zhong T, Wang Z, Meng X et al. (2018). Caspase-3 inhibition attenuates the cytopathic effects of EV71 infection. Frontiers in Microbiology 9: 1402. DOI 10.3389/ fmicb.2018.00817. 
Van Den Berg E, Bal SM, Kuipers MT, Matute-Bello G, Lutter R et al. (2015). The caspase inhibitor zVAD increases lung inflammation in pneumovirus infection in mice. Physiological Reports 3: e12332. DOI 10.14814/phy2.12332.

Wang C, Zhang Y, Han L, Guo L, Zhong H, Wang J (2017a). Hemin ameliorates influenza pneumonia by attenuating lung injury and regulating the immune response. International Journal of Antimicrobial Agents 49: 45-52. DOI 10.1016/j. ijantimicag.2016.09.030.

Wang H, Li K, Ma L, Wu S, Hu J et al. (2017b). Berberine inhibits enterovirus 71 replication by downregulating the MEK/ ERK signaling pathway and autophagy. Virology Journal 14: 382. DOI 10.1186/s12985-016-0674-4.

Wu D, Zhou WY, Lin XT, Fang L, Xie CM (2019). Bufalin induces apoptosis via mitochondrial ROS-mediated caspase-3 activation in HCT-116 and SW620 human colon cancer cells. Drug and Chemical Toxicology 42: 444-450. DOI 10.1080/01480545.2018.1512611.

Xiao Y, Zhou J, Zhang H, Ding C, Shi P (2018). Epidemiological and aetiological characteristics of hand, foot and mouth disease cases 2011-2017 in Yixing, China. Infectious Diseases 50: 859-861. DOI 10.1080/23744235.2018.1493217.
Xing W, Liao Q, Viboud C, Zhang J, Sun J et al. (2014). Hand, foot, and mouth disease in China, 2008-12: An epidemiological study. Lancet Infectious Diseases 14: 308-318. DOI 10.1016/ S1473-3099(13)70342-6.

Xu T, Lin Z, Wang C, Li Y, Xia Y et al. (2019). Heat shock protein 70 as a supplementary receptor facilitates enterovirus 71 infections in vitro. Microbial Pathogenesis 128: 106-111. DOI 10.1016/j.micpath.2018.12.032.

Zeng S, Meng X, Huang Q, Lei N, Zeng L et al. (2019). Spiramycin and azithromycin, safe for administration to children, exert antiviral activity against enterovirus A71 in vitro and in vivo. International Journal of Antimicrobial Agents 53: 362-369. DOI 10.1016/j.ijantimicag.2018.12.009.

Zhao MQ, Wang LH, Lian GW, Lin ZF, Li YH et al. (2019). Characterization of lymphocyte subsets in peripheral blood cells of children with EV71 infection. Journal of Microbiology, Immunology and Infection 53: 705-714. DOI 10.1016/j.jmii.2019.03.001.

Zhu B, Xu T, Lin Z, Wang C, Li Y et al. (2017). Recombinant heat shock protein 78 enhances enterovirus 71 propagation in Vero cells and is induced in SK-N-SH cells during the infection. Archives of Virology 162: 1649-1660. DOI 10.1007/s00705-017-3287-3. 